\title{
Temperature and Coverage Dependent Quasi-reversible Two-photon Photoemission of 1-phenyl-1-propyne on $\mathrm{Cu}(111)$
}

\author{
Youngku Sohn, ${ }^{\dagger, t, *}$ Wei Wei, ${ }^{\ddagger, a}$ Weixin Huang, ${ }^{\S}$ and John M. White ${ }^{\ddagger, b}$ \\ 'Department of Chemistry, Yeungnam University, Gyeongsan, Gyeongbuk 712-749, Korea. ${ }^{*}$ E-mail: youngkusohn@ynu.ac.kr \\ Department of Chemistry and Biochemistry, Center for Materials Chemistry, University of Texas at Austin, TX 78712 \\ ${ }^{\S}$ Hefei National Laboratory for Physical Sciences at the microscale and Department of Chemical Physics, University of Science \\ and Technology of China, Hefei 230026, China \\ Received January 17, 2011, Accepted May 6, 2011
}

\begin{abstract}
A temperature- and coverage-dependant quasi-reversible change in two-photon photoemission (2PPE) of chemisorbed 1-phenyl-1-propyne (PP) on $\mathrm{Cu}(111)$ is reported. For $\mathrm{PP}$ on $\mathrm{Cu}(111)$ at $300 \mathrm{~K}$ probed at a photon energy of $4.13 \mathrm{eV}$, two broad peaks of comparable intensity show final state energies of 7.25 and $7.75 \mathrm{eV}$ above the Fermi level. The former peak could be assigned to the first image potential state (IS, $n=1)$ and/or unoccupied molecular orbital (UMO), located at $3.1 \mathrm{eV}$ above the Fermi level. The latter is plausibly attributed to a mix of unoccupied higher-order IS (and/or UMO) and occupied surface state (SS) of $\mathrm{Cu}(111)$. With decreasing the temperature, the former 2PPE peak shows a shift in position by about $0.2 \mathrm{eV}$, and the latter exhibits a dramatic increase in intensity. In the system, intermolecular interactions (and/or order-disorder transition) of PP and substrate lattice temperature may play a significant role in change in photoexcitation lifetime (or excitation cross-section), and the unoccupied molecular orbital (UMO)-metal (IS) charge transfer coupling. Our unique $2 \mathrm{PPE}$ results provide a deeper insight for understanding photoexcitation charge transfer with temperature in an organic molecule/metal system.
\end{abstract}

Key Words : Two-photon photoemission spectroscopy, Image potential state, Surface state, Photoexcitation charge transfer, 1-Phenyl-1-propyne (PP)

\section{Introduction}

The aligned energy levels at molecule/metal interfaces and the relevant charge transfer processes are critically important to understand such as performance of molecular electronic devices and surface photochemical reactions. ${ }^{1-6}$ For a simple molecular electronic device consisting of a molecule sandwiching between two metal electrodes, a basic operation concept is a charge flow from one to the other electrode through the sandwiched molecule. ${ }^{1,2}$ In the device, the electron and hole barrier heights are one of the most important factors determining efficiency. The binding of an anchor functional group such as thiol (-SH) with the metal electrode is directly related with the interfacial energy levels and electronic couplings. ${ }^{2}$ For understanding photo-induced charge transfer chemical reactions, an electronic coupling between the unoccupied orbitals of molecules and metal substrates needs to be understood. ${ }^{1}$

Two-photon photoemission spectroscopy (2PPE) has been widely employed to investigate the interfacial energy levels and the electronic couplings at molecules/metal, however, the metal-UMO resonance has been debated because the resonance is absent in most cases. ${ }^{1,2,-9}$ Motivated by this, we further delve into this issue by investigating the 1-phenyl-1-

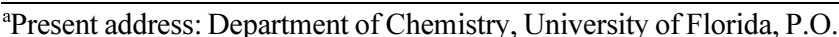
Box 117200 Gainesville, FL 32611-7200, United States

${ }^{\mathrm{b}}$ Deceased August 31, 2007
propyne/Cu(111) system by means of $2 \mathrm{PPE}$, ultraviolet photoelectron spectroscopy (UPS) and temperature-programmed desorption (TPD) spectroscopy.

\section{Experimental Section}

The experiments were performed in an ultrahigh-vacuum chamber with a base pressure of less than $5 \times 10^{-10}$ Torr, equipped with a time-of-flight (TOF) mass spectroscopy, Xray photoelectron spectroscopy (XPS), UPS, Auger electron spectrometer, and a time-of-flight electron energy analyzer for 2PPE spectroscopy. The $\mathrm{Cu}$ (111) (13 mm diameter and $2.5 \mathrm{~mm}$ thick, MaTecK) single crystal was cleaned by several cycles of $\mathrm{Ar}+$ ion sputtering and annealing until no contaminant was detected by XPS or Auger electron spectroscopy (AES). The 1-phenyl-1-propyne (Acros, 98\%) was degassed by several freeze-pump-thaw cycles using liquid nitrogen. The molecule was dosed on the $\mathrm{Cu}(111)$ surface at $100 \mathrm{~K}$ or a selected temperature using a leak valve. Time-offlight (TOF) temperature programmed desorption (TPD) spectra were taken at a temperature ramp rate of $2 \mathrm{~K} / \mathrm{s}$. For 2PPE, we utilized a tunable Spectra-Physics MOPO/FDO900 laser system pumped by a Quanta-Ray 170-10 Nd-YAG laser, and the photon pulses $(7 \mathrm{~ns})$ were incident at $45^{\circ} .{ }^{10}$

\section{Results and Discussion}

On the basis of temperature programmed desorption (TPD) 
profiles of 1-phenyl-1-propyne on $\mathrm{Cu}(111)$ dosed at 100 , 300 and $340 \mathrm{~K}$, 1-phenyl-1-propyne (PP) molecularly adsorbs and desorbs without detectable dissociation. ${ }^{11}$ For the first-layer chemisorbed PP, a minor and a major peak are observed at 320 and $390 \mathrm{~K}$, respectively, with an integrated intensity ratio of $0.15 .^{11}$ The major peak has been assigned to a cross-bridged $-\mathrm{C}=\mathrm{C}$ - geometry, where the $s p$ hybridization of carbon of acetylene group changes to $s p^{2}$. The minor peak has been assigned to vertically aligned vinilyidene-type structure.

Figure 1 shows the 2PPE spectra of 1.0 ML PP on $\mathrm{Cu}(111)$ dosed at $300 \mathrm{~K}$, measured with decreasing the substrate temperature. In the spectra, three major photoemission characteristics are revealed. First, in the 2PPE spectra taken at $300 \mathrm{~K}$, two broad peaks are clearly seen above $7 \mathrm{eV}$; one peak at $\sim 7.25 \mathrm{eV}$ varies linearly with photon energy while the other peak at $\sim 7.8 \mathrm{eV}$ exhibits a linear fit slope of slope $=$ $1.5 \pm 0.25$. The details of the slopes and the peak assignment are described in elsewhere. ${ }^{11}$ Briefly, in 2PPE process, if the variation is 1 the photoemission peak is due to a photoionization from an unoccupied state, corresponding to the former peak. The former peak (slope $=1$ ) is then assigned to an unoccupied state. ${ }^{1,2}$ The peak could be due to the first image potential state (IS, $\mathrm{n}=1$ ). Considering the probe photon energy of $4.13 \mathrm{eV}$, the unoccupied state is located at $\sim 1.0 \mathrm{eV}$ below the vacuum level (or $3.1 \mathrm{eV}$ above the Fermi level). This value is fairly close to the value of IS $(n=1)$ for clean
$\mathrm{Cu}(111)$ reported in the literature. ${ }^{12,13}$ For $1 \mathrm{ML}$ naphthalene/ $\mathrm{Cu}(111)$, Wang et al., found an unoccupied state located at $1.1 \mathrm{eV}$ below the vacuum level (or $3.1 \mathrm{eV}$ above the Fermi level). ${ }^{14}$ This is in very good agreement with our result. They showed that the unoccupied state behaves like an image state. In this paper, we will tentatively assign the unoccupied state peak to IS $(n=1)$ and/ or unoccupied molecular orbital (UMO). For the latter peak, unfortunately, the peak could not be well-resolved using our experimental (photon energy) setup. The slope of 1.5 is neither solely due to an unoccupied state nor an occupied state. The direct two-photon photoemission from an occupied state exhibits a slope of 2 . This could suggest that the slope of 1.5 is a mixed state originated from both unoccupied (slope $=1$ ) and occupied (slope=2) states. We attribute the slope $=2$ to $\mathrm{Cu}$ surface state (SS). The slope $=1$ could be due to higher-lying image potential state (IS) ${ }^{11} \mathrm{UMO}$, or a mixed IS/UMO state. In addition to the two broad peaks, the peak at $\sim 6.1 \mathrm{eV}$ shows a slope of 2 with photon energy, and is assigned to the highest occupied molecular orbital (HOMO) and $\mathrm{Cu} d$-band. ${ }^{10}$

The second main observation in Figure 1 is that as the temperature is lowered the intensity of the latter peak (IS/ $\mathrm{UMO}+\mathrm{SS}$ ) dramatically increases accompanying the narrowness of the peak. While the HOMO/d-band and the $\operatorname{IS}(n=1) /$ UMO peaks show slight decreases in intensity, as seen in Figure 1. The former IS(n=1)/UMO peak shows a shift to the higher kinetic energy side, a decrease in intensity, and a
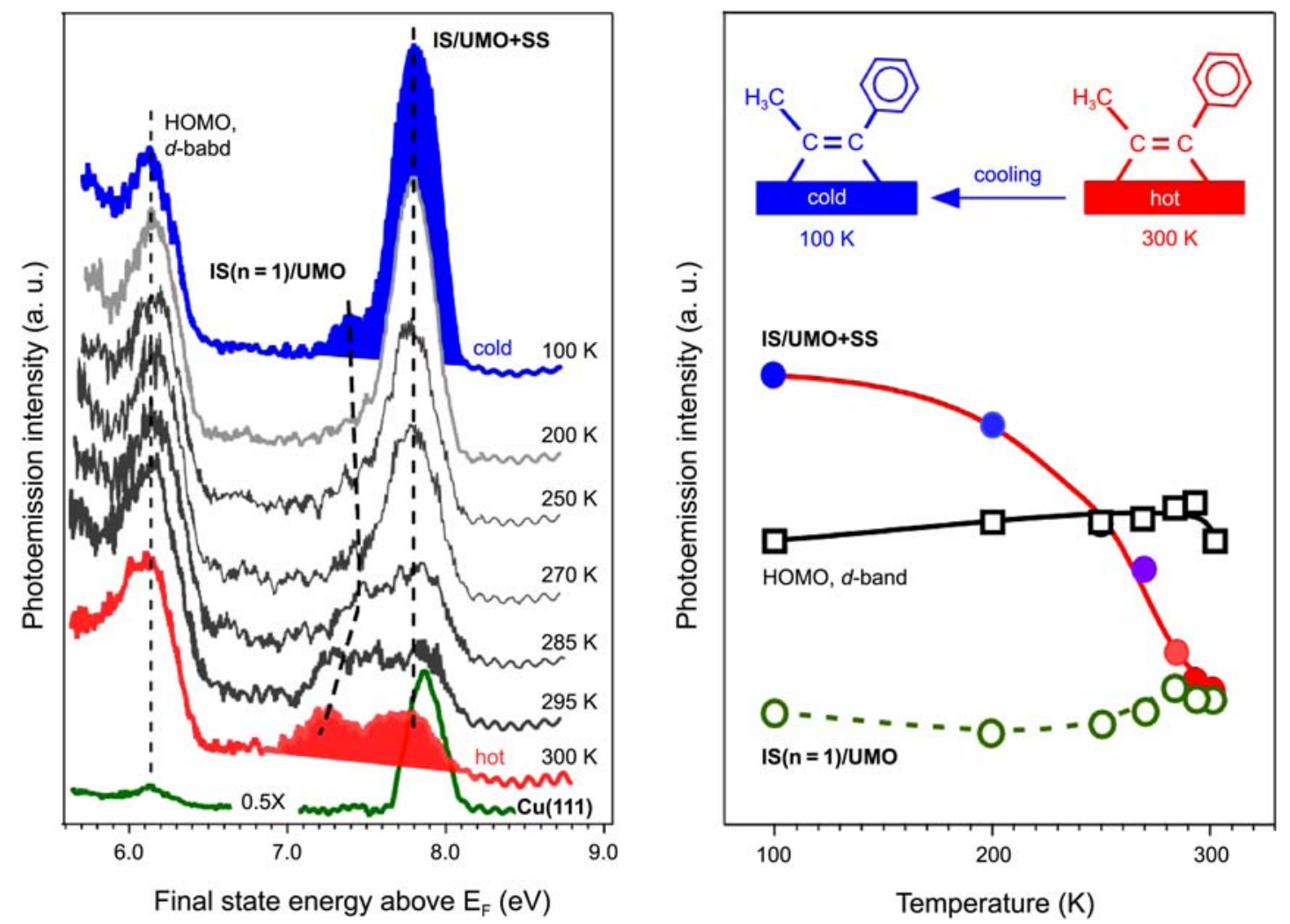

Figure 1. 2PPE spectra taken with lowering temperature from 300 to $100 \mathrm{~K}$ for $1.0 \mathrm{ML} \mathrm{PP}$ in $\mathrm{Cu}(111)$ dosed at $300 \mathrm{~K}$, at a pump-probe photon energy of $4.13 \mathrm{eV}$, including the $2 \mathrm{PPE}$ of bare $\mathrm{Cu}(111)$ at $300 \mathrm{~K}$. IS=image potential state; UMO=unoccupied molecular orbital; $\mathrm{SS}=$ surface state. The laser power was fixed during the measurements. Right figure shows the changes in the peak intensities with temperature. 
narrowness of the peak. In the 270-200 $\mathrm{K}$ region, the $\mathrm{IS}(\mathrm{n}=1) / \mathrm{UMO}$ peak is not clearly revealed, which has been reproducibly observed. It appears that the former and the latter peaks merge to a single peak. The IS( $n=1) / \mathrm{UMO}$ peak may be shifted further and buried in the IS/UMO+SS peak. In the temperature below $200 \mathrm{~K}$, the IS(n=1)/UMO peak obviously reappears, and looks re-separated from the IS/ $\mathrm{UMO}+\mathrm{SS}$ peak. Upon cooling to $100 \mathrm{~K}$, the state is positioned at $\sim 0.85 \mathrm{eV}$ below the vacuum level, $0.15 \mathrm{eV}$ shift towards the vacuum level. We assume that a change in $\mathrm{Cu}$ lattice with temperature could lead the changes in spectral shape and position. ${ }^{15}$ We also do not exclude a wave function mixing between the metal and PP involving the photoemission from UMO. For physisorbed molecules such as benzene and naphthalene on metal substrates, the electronic coupling between metal and UMO is weak, and the UMO peak is commonly not seen in the 2PPE spectra. ${ }^{1,2,7}$ It should be emphasized here that the $2 \mathrm{PPE}$ of bare $\mathrm{Cu}(111)$ shows no critical change in intensity with temperature. Therefore, the observed reversible changes must involve chemisorbed PP molecules on $\mathrm{Cu}(111)$.

The third is that the 2PPE spectra quasi-reversibly change with temperature as seen in Figure 2(a). The enhanced IS/ $\mathrm{UMO}+\mathrm{SS}$ peak attenuates upon re-heating to 260 and $300 \mathrm{~K}$, and re-grows by re-cooling to $100 \mathrm{~K}$. And, the $\mathrm{IS}(\mathrm{n}=1) /$ UMO peak oppositely shows the same phenomenon. For multilayer PP on $\mathrm{Cu}(111)$ at $100 \mathrm{~K}$, the $2 \mathrm{PPE}$ spectrum is very similar to that taken at $300 \mathrm{~K}$. To check the effect of the vertically aligned minor chemisorbed $\mathrm{PP}^{11}$ on the photoemission intensity with temperature, we dosed $\mathrm{PP}$ on $\mathrm{Cu}(111)$ at $340 \mathrm{~K}$ to remove the minor PP, and took $2 \mathrm{PPE}$ spectra with decreasing temperature. As mentioned above, the desorption temperature of the minor PP is at $320 \mathrm{~K}$, and the surface coverage of $\mathrm{PP}$ is about $0.8 \mathrm{ML}$ after dosing at 340 $\mathrm{K}$. The 2PPE spectra in Figure 2(b) also show the very similar change in intensity as the spectra in Figure 1. The intensity of the latter peak (IS/UMO+SS) at $100 \mathrm{~K}$ is enhanced by $10 \times$, compared to that at $340 \mathrm{~K}$. The $\mathrm{IS}(\mathrm{n}=1) /$ UMO peak almost disappears at 290 and $270 \mathrm{~K}$, but reappears at lower temperatures (160 and $100 \mathrm{~K})$. From the result, we conclude that the vertically aligned $\mathrm{PP}$ is not importantly involved in the enhancement of IS/UMO+SS peak, and the shift in IS(n=1)/UMO peak. To check the influence of PP coverage on the photoemission intensity, we further reduced the coverage of $\mathrm{PP}$ on $\mathrm{Cu}(111)$ by dosing at a higher temperature of $380 \mathrm{~K}$, corresponding to a coverage of about 0.5 ML. Interestingly, the 2PPE spectra show no critical change with decreasing temperature to $100 \mathrm{~K}$ (Figure 2(c)). In addition, the latter IS/UMO+SS peak becomes weaker than the former IS $(n=1) / \mathrm{UMO}$ peak. This indicates that the $2 \mathrm{PPE}$ of $\mathrm{PP} / \mathrm{Cu}(111)$ is a coverage-dependant. In other words, intermolecular interactions may play an impor-
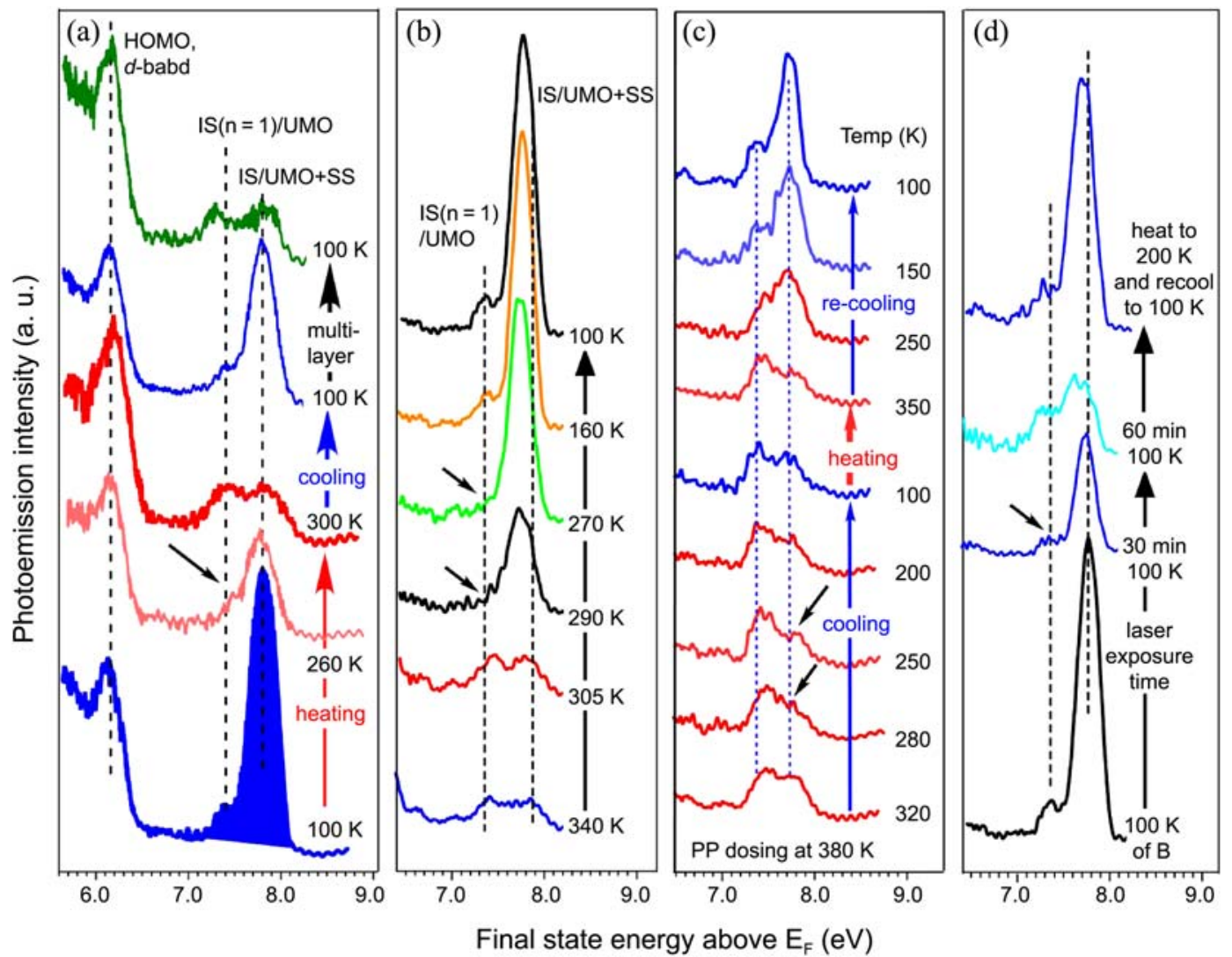

Figure 2. (a) 2PPE spectra upon reheating to 260 and $300 \mathrm{~K}$, and cooling back to $100 \mathrm{~K}$. 2PPE spectrum upon further dosing to form multilayer. (b) 2PPE spectra for PP dosed at $340 \mathrm{~K}$ with decreasing temperature. (c) 2PPE spectra of PP on Cu(111) dosed at $380 \mathrm{~K}$ with cooling, heating and recooling the substrate. (d) 2PPE spectra (at $100 \mathrm{~K}$ ) after exposing the incident laser for 30 and 60 min, and after reheating to $200 \mathrm{~K}$ followed by cooling to $100 \mathrm{~K}$. 


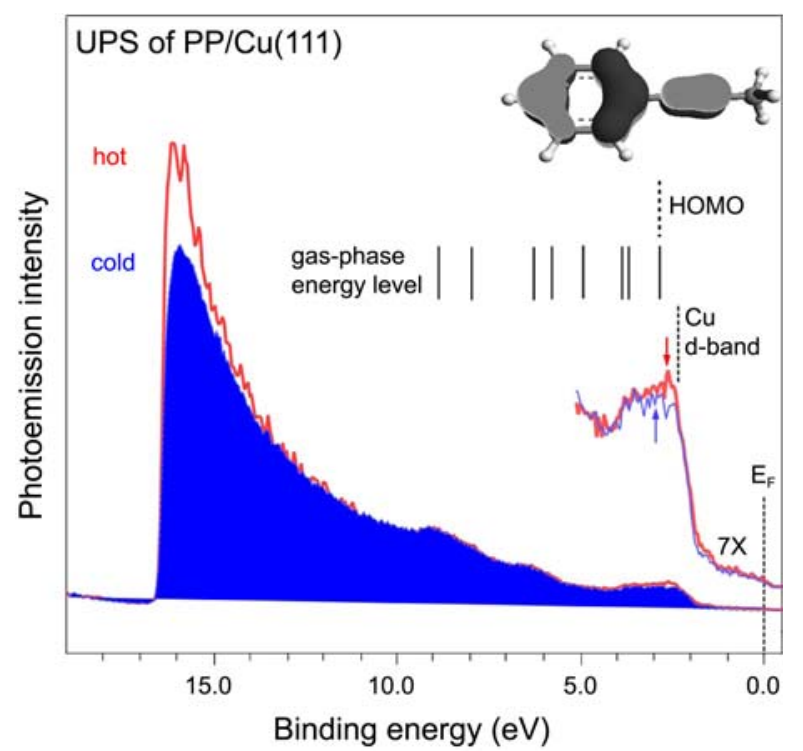

Figure 3. UPS spectra $(\mathrm{He} \mathbf{I}, \hbar \omega=21.2 \mathrm{eV})$ of 1.0 ML PP on $\mathrm{Cu}(111)$ dosed at $300 \mathrm{~K}$, taken at 300 (hot) and $100 \mathrm{~K}$ (cold). Upper right shows the corresponding spectra after $7 \times$. Binding energy is referenced to the Fermi level $\left(\mathrm{E}_{\mathrm{F}}=0.0 \mathrm{eV}\right)$. Theoretically calculated gas-phase energy levels and HOMO of PP are displayed for reference. ${ }^{11}$

tant role in change in the $2 \mathrm{PPE}$ with temperature. The intensity of the latter peak at $100 \mathrm{~K}$ could be more or less enhanced, but not as much as the peak in Figure 2(b), by reheating to $350 \mathrm{~K}$ followed by re-cooling to $100 \mathrm{~K}$. The $2 \mathrm{PPE}$ is also influenced by laser irradiation. The IS/UMO+SS peak intensity deteriorates with increasing laser exposure time (Figure 2(d)), but the intensity could be recovered by reheating and cooling. In Figure 2(d), the peak intensity is recovered after reheating to $200 \mathrm{~K}$ followed by cooling to $100 \mathrm{~K}$.

Figure 3 shows the ultraviolet one-photon photoemission spectra taken at 300 and $100 \mathrm{~K}$ for the fully saturated $1.0 \mathrm{ML}$ PP on $\mathrm{Cu}(111)$. For the 1.0 ML PP on $\mathrm{Cu}(111)$, the work function is lowered by $0.85 \mathrm{eV}$, referenced to the work function $(\Phi=4.95 \mathrm{eV})$ of bare $\mathrm{Cu}(111) .{ }^{11}$ Unlike the $2 \mathrm{PPE}$ profiles, there is no notable change in photoemission peak profiles with temperature. In addition, the exactly same low energy secondary electron cutoff indicates no change in work function (or no change in molecular dipole alignment) with temperature. Because of the same broad peak positions $(\mathrm{BE}=0$ to $10 \mathrm{eV})$ and no change in work function, we ruled out the scenario of changing from the state of chemisorption to that of physisorption with lowering temperature. ${ }^{8}$ One may argue that although the change in UPS is not significant as $2 \mathrm{PPE}$, there is a slight spectral change (marked by arrows) in the HOMO position between 0.0 and $5.0 \mathrm{eV}$ ( $7 \times$ expanded spectra). This could be related with a slight change in chemical interaction between $\mathrm{PP}$ and $\mathrm{Cu} d$-band, ${ }^{12}$ consequently leading a dramatic change in 2PPE.

Then, why does the dramatic change in 2PPE peak occur without disturbing the main chemical bonding? The dramatic enhancement could be due to a resonance excitation between the surface and image potential states. When a photon energy $(\hbar \omega)$ tunes to the energy gap the transition probability from the SS to the IS increases considerably, leading to an overlap and a drastic enhancement of the photoemission peak. ${ }^{16}$ However, this may not be the major reason because the IS/UMO+SS peak probed at lower photon energies (e.g., $3.76 \mathrm{eV}$, an off-resonance condition) is also dramatically enhanced by about the same fold increase, compared to the spectra taken at $300 \mathrm{~K}$. In addition, if the resonance excitation is the major reason, the 2PPE enhancement should also occur irrespective of temperature and coverage. But this is not consistent with our results. Image state lifetime and dephasing rate influenced by lattice temperature are plausibly considered.$^{15,17}$ For clean $\mathrm{Cu}(111)$, Wolf and coworkers first observed that IS and SS peaks shift with temperature. ${ }^{15}$ They explained the temperature dependent 2PPE by lifetime and dephasing rate. The former IS( $n=1) /$ UMO peak in Figure 1 could be explained by this.

For an organic molecule on metal substrate, the 2PPE intensity (especially the latter IS/UMO+SS peak) more drastically changes with temperature, and our 2PPE is the first observation. Then, plausible scenarios can be suggested on the basis our results as discussed above: intermolecular interactions (or order-disorder transition [18]) of adsorbate and the $\mathrm{Cu}$ lattice temperature could change the $2 \mathrm{PPE}^{1}$ without disturbing the interface dipole and the main chemical bonding. Schöll et al. have shown that the interfacial bonding between an organic molecule and metal could be changed with temperature by order-disorder transition. ${ }^{18}$ Consequently, with decreasing temperature, the binding energies of occupied and unoccupied states vary to change UMOmetal (IS) charge transfer coupling (or excitation crosssection and photoexcitation lifetime), leading to enhancing the 2PPE intensity.

\section{Summary}

In summary, for an organic molecule/metal system we have first observed that the 2PPE characteristics of 1phenyl-1-propyne on $\mathrm{Cu}(111)$ quasi-reversibly changes with temperature, without a change in $\mathrm{PP}-\mathrm{Cu}(111)$ chemical bonding. The change in UPS spectrum with temperature is not significant as the corresponding 2PPE spectrum. For bare $\mathrm{Cu}(111)$, no significant change in 2PPE was found with temperature using our experimental setup. Therefore, the PP molecules are must be involved in the temperature- and coverage-dependant quasi-reversible change in 2PPE. In the 2PPE spectrum, two broad peaks with final state energies of 7.25 and $7.75 \mathrm{eV}$ above the Fermi level are seen. The former peak is assigned to unoccupied IS( $n=1) / \mathrm{UMO}$ state located at $\sim 1.0 \mathrm{eV}$ below the vacuum level (or $3.1 \mathrm{eV}$ above the Fermi level). The latter peak is attributed to a mix of unoccupied IS/UMO and occupied SS states. With lowering the temperature, the IS(n=1)/UMO peak shifts towards the vacuum level, and latter peak shows a dramatic increase in intensity. We have explained the 2PPE results by changes in photoexcitation lifetime (excitation cross-section), dephas- 
ing rates, and the unoccupied molecular orbital (UMO)metal (IS) charge transfer coupling which are plausibly influenced by the intermolecular interactions (or orderdisorder transition) of adsorbate and $\mathrm{Cu}$ lattice temperature. Although some are still under question, and requires more supporting experimental evidences, our unique temperatureand coverage-dependent $2 \mathrm{PPE}$ results provide a new insight for deeper understanding of photoexcitation charge transfer process between an adsorbate organic molecule and a metal surface.

Acknowledgments. This work was financially supported by the Center for Materials Chemistry at the University of Texas at Austin, Robert A. Welch Foundation (Grant F0032), and National Science Foundation (CHE 0412609). W.H. gratefully acknowledges the financial support from National Natural Science Foundation of China (grant 20503027) and the Hundred Talent program of Chinese Academy of Sciences.

\section{References}

1. Zhu, X.-Y. Annu. Rev. Phys. Chem. 2002, 53, 221.

2. Zhu, X.-Y. Surf. Sci. Rep. 2004, 56, 1.
3. Ryu, S.; Chang, J.; Kwon, H.; Kim, S. K. J. Am. Chem. Soc. 2006, $128,3500$.

4. Gudde, J.; Berthold, W.; Hofer, U. Chem. Rev. 2006, 106, 4261.

5. Petek, H.; Ogawa, S. Prog. Surf. Sci. 1997, 56, 239.

6. Harris, C. B.; Ge, N.-H.; Lingle, R. L., Jr.; McNeill, J. D.; Wong, C. M. Annu. Rev. Phys. Chem. 1997, 48, 711.

7. Gaffney, K. J.; Miller, A. D.; Harris, C. B. J. Phys Chem. B. 2001, $105,9031$.

8. Lindstrom, C. D.; Muntwiler, M.; Zhu, X.-Y. J. Phys. Chem. B. 2005, 109, 21492.

9. Wolf, M.; Hotzel, A.; Knoesel, E.; Velic, D. J. M. Phys. Rev. B 1999, 59, 5926.

10. Wei, W.; Zhao, W.; White, J. M. J. Am. Chem. Soc. 2004, 126, 16340.

11. Sohn, Y.; Wei, W.; White, J. M. Langmuir 2007, 23, 12185.

12. Yamada, T.; Shibuta, M.; Ami, Y.; Takano, Y.; Nonaka, A.; Miyakubo, K.; Munakata, T. J. Phys. Chem. C 2010, 114, 13334.

13. Giesen, K.; Hage, F.; Himpsel, F. J.; Riess, H. J.; Steinman, W. Phys. Rev. B 1986, 33, 5241.

14. Wang, H.; Dutton, G.; Zhu, X.-Y. J. Phys. Chem. B 2000, 104, 10332.

15. Knoesel, E.; Hotzel, A.; Wolf, M. J. Electron Spectrosc. Relat. Phenom. 1998, 88, 577 .

16. Steinmann, W. Phys. Stat. Sol (b) 1995, 192, 339.

17. Fauster, T.; Weinelt, M.; Hofer, U. Prog. Surf. Sci. 2007, 82, 224.

18. Schöll, A.; Kilian1, L.; Zou, Y.; Ziroff1, J.; Hame, S.; Reinert, F.; Umbach, E.; Fink, R. H. Science 2010, 329, 303. 